\title{
Low noise design experience for the SKADS/BEST demonstrator ${ }^{\star}$
}

\author{
F. Perini
}

\begin{abstract}
I.N.A.F.-I.R.A., Via Fiorentina 3508/B, Medicina (BO), Italy
email: f.perini@ira.inaf.it

Abstract. The experience gained in both the design and testing of low noise amplifiers for the SKADS/BEST demonstrator, is here described. Different architectures (single ended, balanced and differential) have been investigated and compared in order to fulfill the BEST goals and, in general, the main SKA related technology requirements: low cost, high performances and reliability. This experience is leading to the design of front ends suitable for low frequency SKA pathfinders and other applications.
\end{abstract}

\section{Introduction}

In order to populate the BEST demonstrator, based on the reinstrumentation of part of the Northern Cross radio telescope, the design of new LNA (Low Noise Amplifier) was necessary. The natural instrument working band is about $5 \mathrm{MHz}$ centred at $408 \mathrm{MHz}$. With proper focal line modifications, $16 \mathrm{MHz}$ centred at $408 \mathrm{MHz}$ (for the N/S arm) and 120-240 MHz (for the E/W arm) have been obtained. The use of commercial LNA was discarded because they are usually noisy and broadband. The latter characteristic makes those devices susceptible to the generation of intermodulation products of received signals even far from the desired band. Moreover, it has been considered important to gain experience in the LNA design in the $\mathrm{f}<1 \mathrm{GHz}$ range, fundamental for SKA AA-lo and AA-hi future developments.

\section{Main design and technological choices}

In order to fulfill both requirements of low cost and high performances, the following design and technological choices have been done:

1. Printed circuit board (PCB) on FR4: very low prototyping cost (milling machine) and moderate production cost;

2. SMD devices: passive elements ( $\mathrm{L}$ and $\mathrm{C}$ ) are smaller and better (i.e. Q factor) than the distributed counterparts, easier active elements mounting (no chip bonding);

3. Hot LNA: cryogenic techniques are too expensive for large array applications and, below $1 \mathrm{GHz}, T_{\text {sky }}$ is not irrelevant as contribute to $T_{\text {sys }}$;

4. Selected active device: AVAGO ATF-54143 (Avago Tech 2008)

- OIP3 $>+32 \mathrm{dBm}$

- $\mathrm{P} 1 \mathrm{~dB}_{\text {out }}>+16 \mathrm{dBm}$

- $\mathrm{NF}<0.2 \mathrm{~dB}$

- Enhancement $\rightarrow$ Single power supply

^ This work was supported by the European Commission Framework Program 6, Project SKADS, Square Kilometre Array Design Studies (SKADS), contract no 011938.

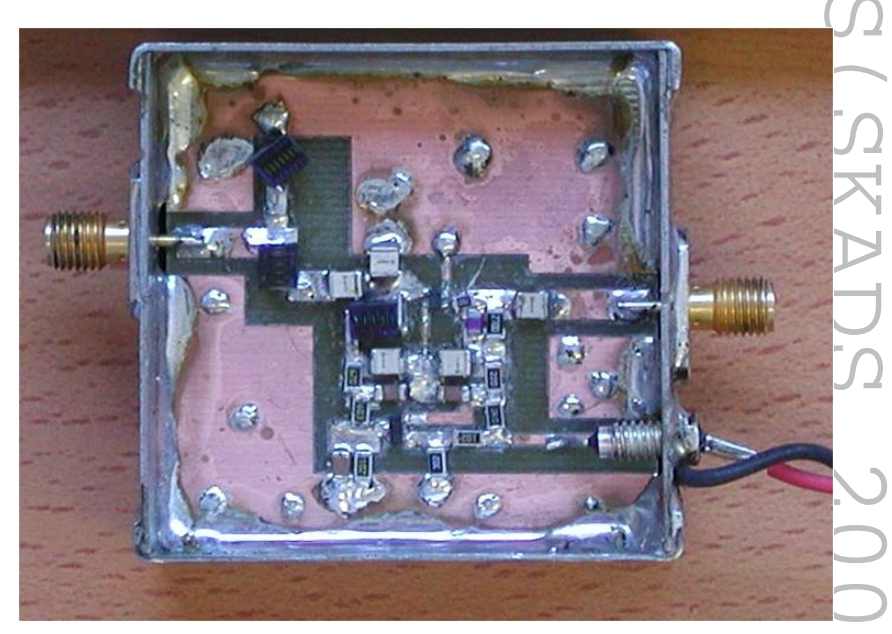

Fig. 1: BEST-1 single ended LNA prototype.

For the LNA designs, AWR(C) Microwave Office has been adopted, both for small and large signals simulations and for layout designs (i.e. gerber files).

\section{LNA for BEST-1 and BEST-2}

BEST is a modular technological demonstrator composed by 3 blocks with different dimensions and characteristics (i.e. collective area, number of receivers, frequency band). BEST-1 has been obtained by installing 4 new receivers on the focal line of a single N/S cylinder. The chosen LNA architecture for the installed front ends, is the standard single ended. The HEMT works at $V_{\mathrm{ds}}=3 \mathrm{~V}$ and $I_{\mathrm{ds}}=60 \mathrm{~mA}$, which represents the optimum compromise between noise and dynamic performances. An active biasing network has been adopted because it provides a means of keeping the quiescent bias point constant over temperature and constant over lot to lot variations in device dc performance. The input matching network is optimised to obtain the best compromise between NF, input matching, stability and rejection of both FM (88-108 MHz) and TV (above $470 \mathrm{MHz}$ ) signals, which intermodulation products could fall in the BEST working band (400-416MHz ). Fig. 1 and Fig. 2 show, respec- 


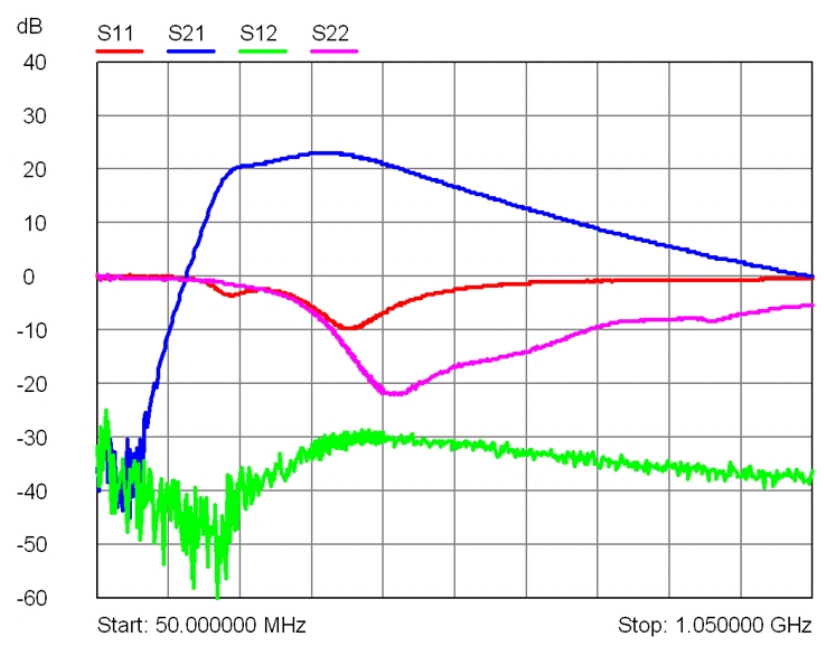

Fig. 2: Single ended S-parameters.

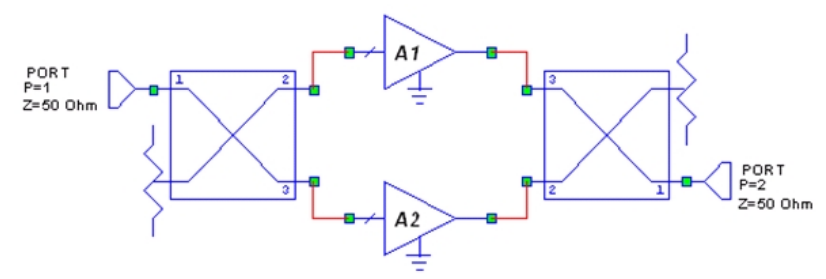

Fig. 3: Balanced amplifier: working principle.

tively, a picture of the realised prototype and the measured Sparameters.

BEST-2 is composed by $8 \mathrm{~N} / \mathrm{S}$ cylindrical concentrators each equipped with 4 receivers, for a total of 32 LNA. The balanced architecture was investigated. In such architecture a pair of equals amplifiers are fed by a couple of $90^{\circ}$ hybrids (see Fig. 3). The input matching network of each amplifier has to be designed only to get the lowest NF possible, simplifying the LNA design. Hybrids take care of the input and output matching. Moreover, since the input and output impedances seen by the active devices (HEMT) are limited by the hybrids, obtaining a stable amplifier is easier. Finally, $\mathrm{P} 1 \mathrm{~dB}_{\text {out }}$ and OIP3 are improved by $3 \mathrm{~dB}$ respect to the single amplifier. A balanced amplifier works well when A1 and A2 and the input and output hybrids are good matched, both in amplitude and phase. A balanced LNA is suitable when, thanks to the best noise matching, the NF reduction of the single stages A1 and A2, respect to the single ended version, is not less than the insertion loss of the input hybrid. Typically, at this working frequency, suitable hybrids are available as cheap SMD transformers, which insertion loss is very low. For BEST-2 the HEMT bias point is again $V_{\mathrm{ds}}=3 \mathrm{~V}$ and $I_{\mathrm{ds}}=60 \mathrm{~mA}$. The hybrids chosen are the HPQ$05 \mathrm{~W}$ by Minicircuits (Fig. 4). As can be noticed comparing both the S-parameters (see both Fig. 2 and Fig. 5) and noise measurements, the balanced LNA allows a very good matching in the working frequency band, with just a little increase of the NF (see Fig. 6). Moreover the balanced LNA works well in a broader band (350-450 MHz) with very interesting

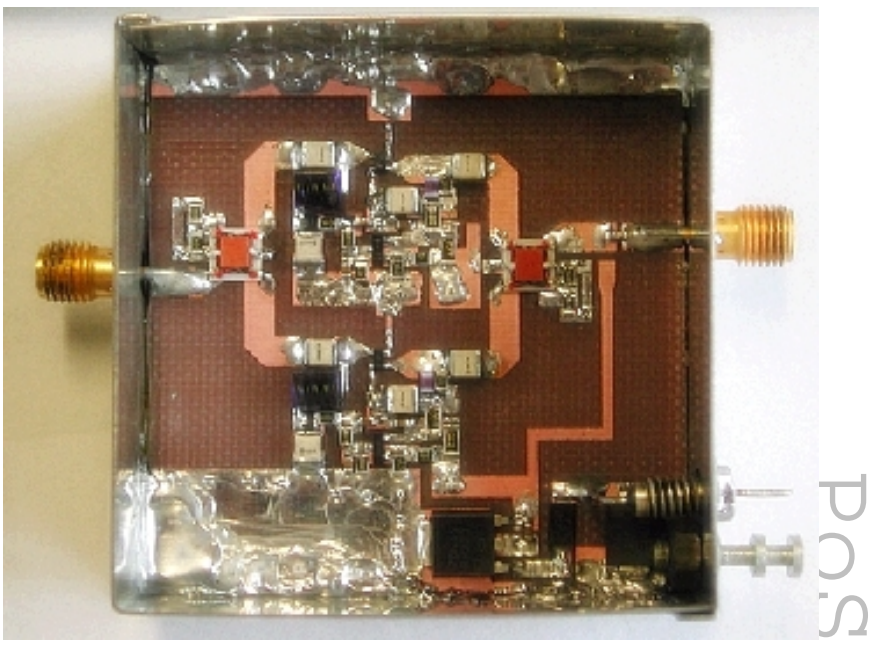

Fig. 4: BEST-2 balanced LNA prototype.

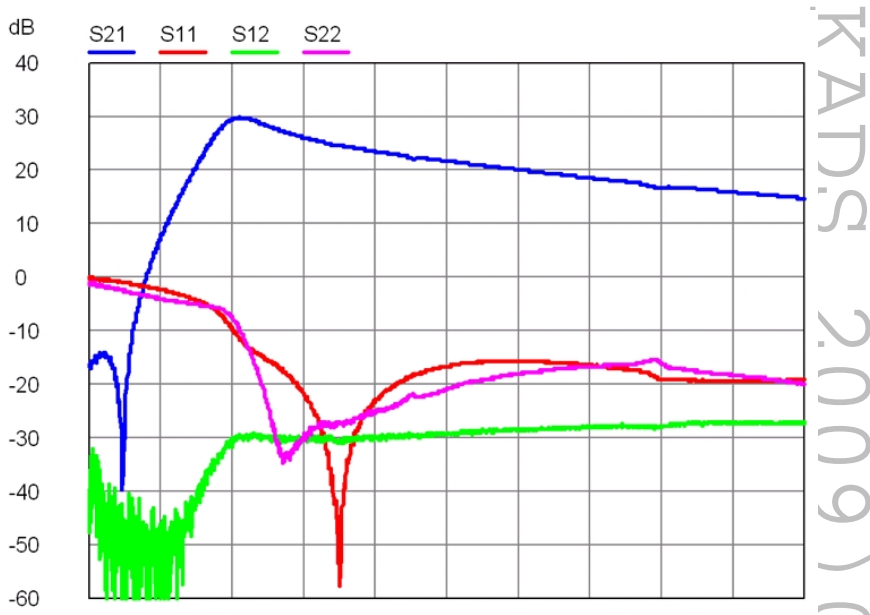

Start: $50.000000 \mathrm{MHz}$

Stop: $1.050000 \mathrm{GHz}$

Fig. 5: Balanced LNA measured S-parameters.

Table 1: Comparison between single ended and balanced LNA at $408 \mathrm{MHz}$

\begin{tabular}{|c|c|c|}
\hline$@ 408 \mathrm{MHz}$ & Single ended & Balanced \\
\hline \hline$|\mathrm{S} 11|[\mathrm{dB}]$ & -10 & -37.5 \\
\hline$|\mathrm{S} 22|[\mathrm{dB}]$ & -17 & -27.1 \\
\hline$|\mathrm{S} 21|[\mathrm{dB}]$ & 22.6 & 24.4 \\
\hline $\mathrm{T}_{\mathrm{n}}[\mathrm{K}]$ & 29.4 & 31.5 \\
\hline $\mathrm{NF}[\mathrm{dB}]$ & 0.42 & 0.45 \\
\hline $\mathrm{OIP} 3[\mathrm{dBm}]$ & +27.3 & +34.5 \\
\hline $\mathrm{P} 1 \mathrm{~dB}_{\text {out }}$ & +12.4 & +21.2 \\
\hline
\end{tabular}

performance: $|\mathrm{S} 11| \&|\mathrm{~S} 22|<-20 \mathrm{~dB}$, OIP3 > +34 dBm and $\mathrm{NF}<0.5 \mathrm{~dB}(380-450 \mathrm{MHz})$. Table 1 summarises the comparison between the single ended and the balanced LNA at the BEST-1 and BEST- 2 central frequency. 


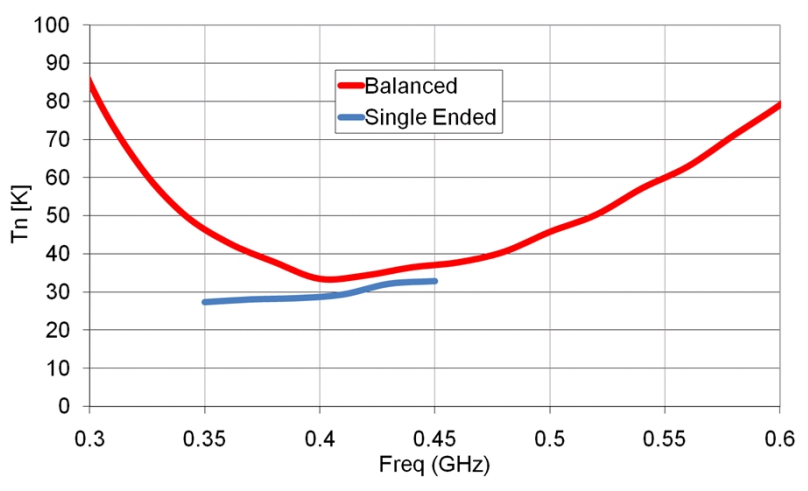

Fig. 6: Noise measurements.

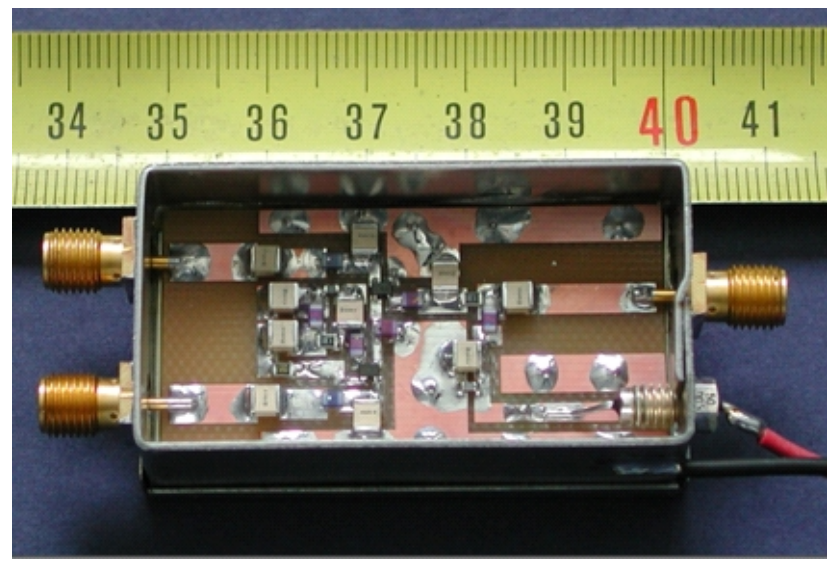

Fig. 7: Differential LNA prototype.

For the BEST system, a differential LNA has been investigated too (see Fig. 7). That configuration could potentially allow to connect directly the LNA to the dipole feed line or, in principle, at each dipole of the Northern Cross radio telescope, avoiding the balun. A cascode configuration has been considered and a commercial MMIC from Motorola (MBC13916) was chosen for the prototype realisation. The differential input of the LNA has been designed at $84 \Omega$, since the $Z_{\text {odd }}$ of the dipole feed line is $42 \Omega$. The simulated NF is quite high (above $1.5 \mathrm{~dB}$ ) because the $N F_{\min }$ of the active device is about $1 \mathrm{~dB}$. Further investigation should be done with other components and/or technologies. Additional experience should also be got in the characterization of differential devices (both $\mathrm{S}$ parameters and noise measures).

\section{BEST-3Io LNA}

BEST-3lo is composed by 18 log-periodic antennas which work in the 120-240 MHz band, installed on one sector of the $\mathrm{E} / \mathrm{W}$ focal line. Due both to the presence of strong signals and the high value of the antenna temperature in the operative band, a balanced architecture LNA has been designed (see Fig. 8). In order to increase the LNA dynamic range, the bias point of the active device has been moved to $V_{\mathrm{ds}}=3 \mathrm{~V}$ and $I_{\mathrm{ds}}=80 \mathrm{~mA}$. A

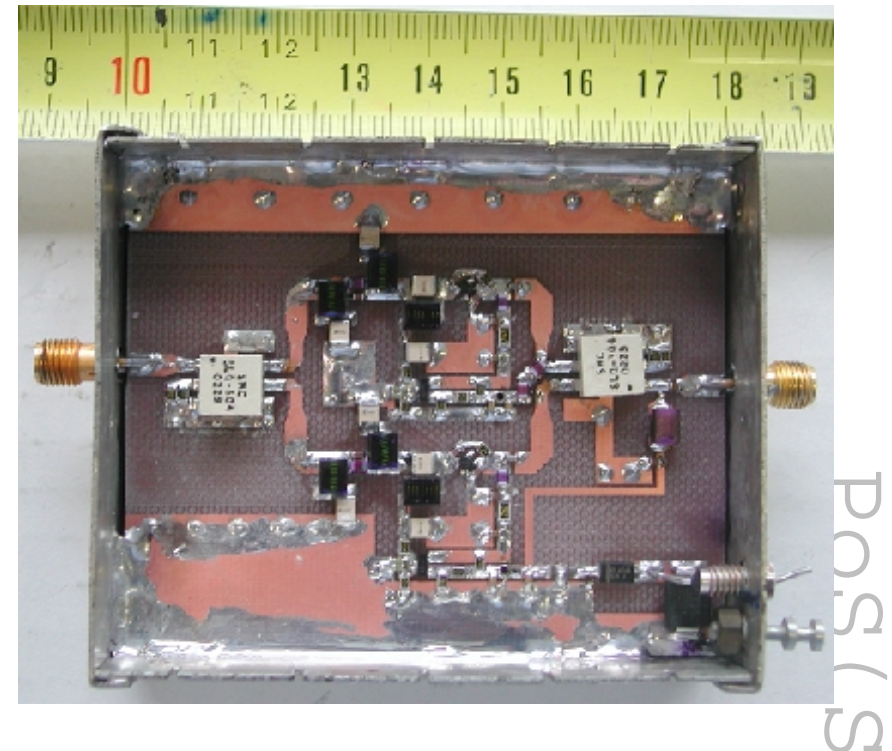

Fig. 8: First generation BEST-3lo LNA prototype.

RC feedback was used to ensure unconditional stability (Avago Tech 2006). To cover the entire frequency band, the SLQ-304 hybrid by Synergy Microwave has been adopted. In order to reject the strongest RFI signals, a FM band stop filter has been designed too and integrated in the LNA. To maintain the best input matching, the filter has been inserted between the outputs of the input hybrid and each active elements. Table 2 summarises the measurements of the prototype.

A second generation low frequency LNA has been designed adopting all COTS devices (see Fig. 9). In particular, the active elements are LNA MMIC by Sirenza (SPF5122Z). The Synergy Microwave hybrid has been replaced by the cheaper Minicircuits ADQ-250-1 (not yet available at the time of the first generation design). The FM and TV signals rejection is obtained by a cascade of a FM band stop filter (Minicircuits BSF-108+), in front of the LNA input, and a low pass filter (Minicircuits RLP-320+) placed between the input hybrids and each LNA. The input hybrid ensures a good isolation between the 2 filters, which allows keeping both good input matching and gain flatness. Each Sirenza LNA is biased at $5 \mathrm{~V}$ and $I_{\mathrm{d}}=90 \mathrm{~mA}$. Fig. 10 and Fig. 11 show, respectively, the noise and the S-parameters measurement.

Table 2: BEST-3lo first prototype measurements

\begin{tabular}{|c|c|c|c|}
\hline Measurement & $@ 130 \mathrm{MHz}$ & @ $200 \mathrm{MHz}$ & @ $250 \mathrm{MHz}$ \\
\hline \hline$|\mathrm{S} 11|[\mathrm{dB}]$ & -21.2 & -25.9 & -22.6 \\
\hline$|\mathrm{S} 22|[\mathrm{dB}]$ & -22.3 & -21.3 & -20.5 \\
\hline$|\mathrm{S} 21|[\mathrm{dB}]$ & 20.5 & 20.9 & 20.7 \\
\hline $\mathrm{NF}[\mathrm{dB}]$ & 3.23 & 1.54 & 1.44 \\
\hline OIP3 $[\mathrm{dBm}]$ & +35.2 & +35.8 & +34.5 \\
\hline P1dB & +21.8 & +23.6 & +22.9 \\
\hline
\end{tabular}




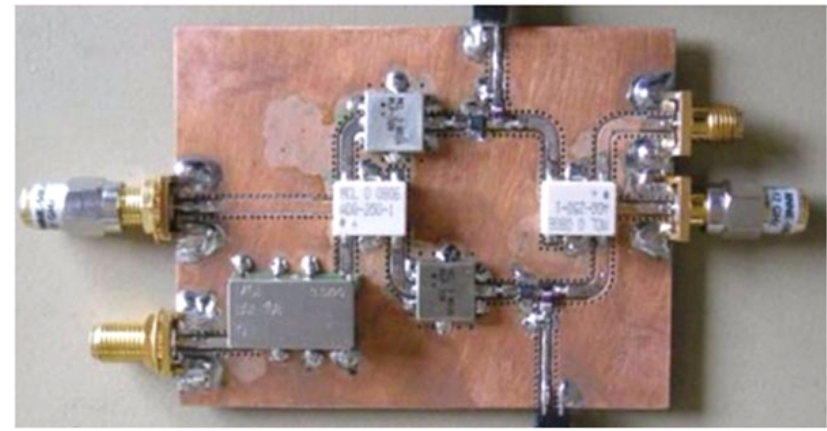

Fig. 9: Second generation BEST-3lo LNA prototype.

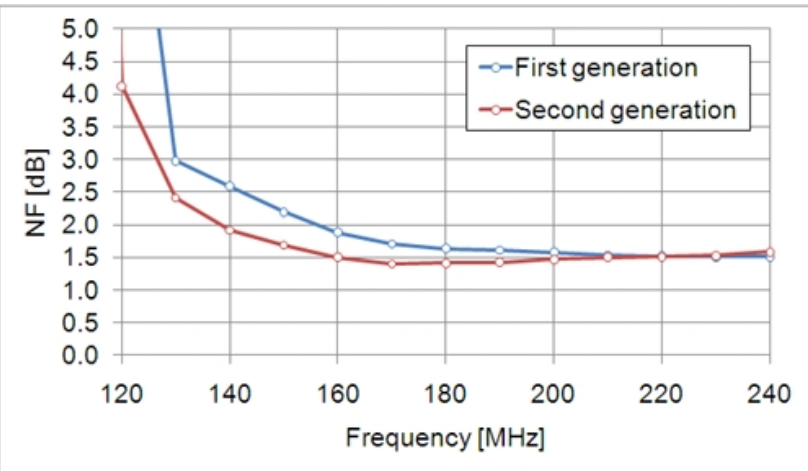

Fig. 10: Noise comparison between the first and second BEST-3lo LNA generations.

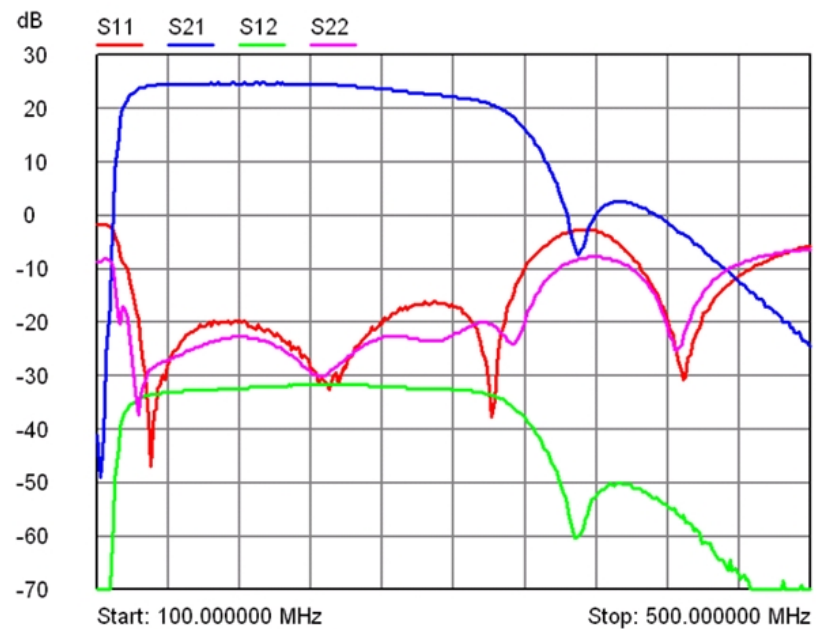

Fig. 11: Second generation BEST-3lo LNA S parameters measurement.

\section{P-band LNA}

As fallout of the experience in the LNA design for BEST, it is evaluating the possibility to adopt the same technology for the P-band LNA for the Sardinia radio telescope (SRT). The frequency band is $305-410 \mathrm{MHz}$ and the LNA must operate at cryogenic temperatures $(20 \mathrm{~K})$. The HEMT bias point is $V_{\mathrm{ds}}=$ $3 \mathrm{~V}$ and $I_{\mathrm{ds}}=28 \mathrm{~mA}$. The active biasing networks has been re-

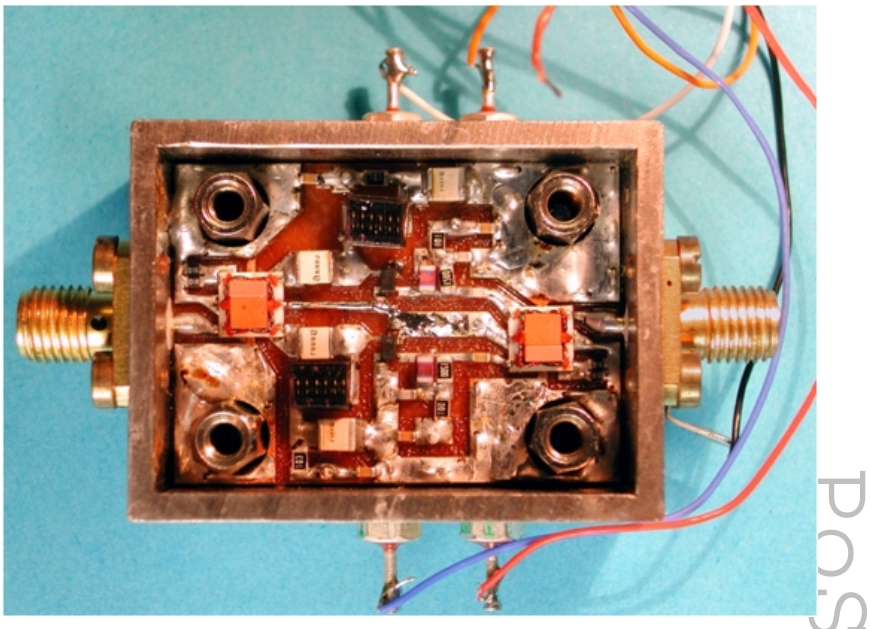

Fig. 12: P-band LNA (305-410 MHz).

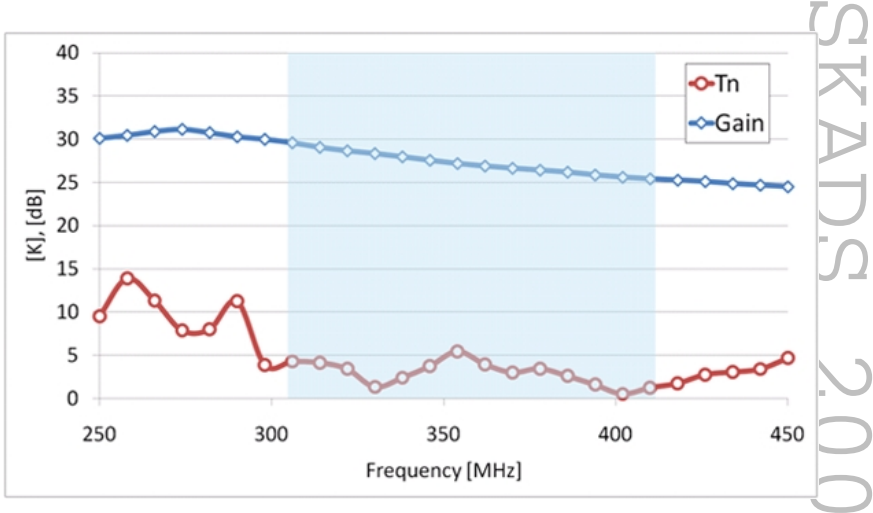

Fig. 13: Preliminary measurements at $20 \mathrm{~K}$.

moved because based on PNP transistor, which does not work at cryogenic temperature. The chosen hybrids are the HPQ-04 by Minicircuits (see Fig. 12). First preliminary results are encouraging (see also Fig. 13): the input and output matching are, respectively, better than $10 \mathrm{~dB}$ and $20 \mathrm{~dB}$ on overall band. The dynamic is good too, with measured $\mathrm{P} 1 \mathrm{~dB}_{\text {out }}>+15 \mathrm{dBm}$ and OIP3 > +26.5 dBm.

\section{Conclusions}

The LNA experience in the framework of the BEST demonstrator allowed us to gain a valuable knowledge both on devices design and characterisation in the frequency range below $\mathrm{f}<1 \mathrm{GHz}$. Such experience has been successfully employed in particular application, as a cryogenic P-band LNA, and could be easily applied in the AAVP program, especially for the AAlo system.

\section{References}

Avago Technologies, "A $100 \mathrm{MHz}$ to $500 \mathrm{MHz}$ Low Noise Feedback Amplifier using ATF-54143," 5989-0852EN, May 8,2006 , available on www . avagotech. com 
Avago Technologies, “ATF-54143 Data sheets," AV020488EN, August 5, 2008, available on www.avagotech. com 